\title{
Construction of Novel Chloroplast Expression Vector and Development of an Efficient Transformation System for the Diatom Phaeodactylum tricornutum
}

\author{
Wei-Hong Xie • Cong-Cong Zhu • Nai-Sheng Zhang • \\ Da-Wei Li • Wei-Dong Yang • Jie-Sheng Liu • \\ Ramalingam Sathishkumar • Hong-Ye Li
}

Received: 1 September 2013 / Accepted: 5 March 2014 /Published online: 26 April 2014

(C) The Author(s) 2014. This article is published with open access at Springerlink.com

\begin{abstract}
Plastids are ideal subcellular hosts for the expression of transgenes and have been successfully used for the production of different biopolymers, therapeutic proteins and industrial enzymes. Phaeodactylum tricornutum is a widely used aquatic feed species. In this study, we focused on developing a high-efficiency plastid expression system for P. tricornutum. In the plastid transformation vector, the site selected for integration was the transcriptionally active intergenic region present between the trnI and trnA genes, located in the IR (inverted repeat) regions of the plastid genome. Initially, a CAT reporter gene (encoding chloramphenicol acetyltransferase) was integrated at this site in the plastid genome. The expression of CAT in the transformed microalgae conferred resistance to the antibiotic chloramphenicol, which enabled growth in the selection media. Overall, the plastid transformation efficiency was found to be approximately one transplastomic colony per 1,000 microalgae cells. Subsequently, a heterologous gene expression cassette for high-level expression of the target gene was created and cloned between the homologous recombination elements. A TA cloning strategy based on the designed $X c m I-X c m I$ sites
\end{abstract}

Wei-Hong Xie and Cong-Cong Zhu contributed equally in this article.

Electronic supplementary material The online version of this article (doi:10.1007/s10126-014-9570-3) contains supplementary material, which is available to authorized users.

W.-H. Xie · C.-C. Zhu • N.-S. Zhang • D.-W. Li • W.-D. Yang J.-S. Liu $\cdot$ H.-Y. Li $(\bowtie)$

Key Laboratory of Eutrophication and Red Tide Prevention of Guangdong Higher Education Institute, Jinan University,

510632 Guangzhou, China

e-mail: thyli@jnu.edu.cn

R. Sathishkumar

Plant Genetic Engineering Laboratory, Department of

Biotechnology, Bharathiar University, Coimbatore, Tamil

Nadu 641046, India could conveniently clone the heterologous gene. An $e G F P$ (green fluorescent protein) reporter gene was used to test the expression level in the plastid system. The relatively highlevel expression of eGFP without codon optimisation in stably transformed microalgae was determined to account for $0.12 \%$ of the total soluble protein. Thus, this study presents the first and convenient plastid gene expression system for diatoms and represents an interesting tool to study diatom plastids.

Keywords Bioreactor · Plastid transformation · Microalga Diatom $\cdot$ Phaeodactylum tricornutum

\section{Introduction}

Marine diatoms are responsible for a high level of productivity and are required to sustain life in the sea. Phaeodactylum tricornutum is an essential phytoplankton used as a live feed in aquaculture, as it is rich in protein and fatty acid contents. P. tricornutum is also rich in EPA (eicosapentaenoic fatty acid), a $\omega-3$ fatty acid that is a valuable nutritional supplement. P. tricornutum is one of the four diatoms for which the genome sequence is available, with the rest three being Thalassiosira pseudonana, Fragilariopsis cylindrus and Pseudo-nitzschia multiseries (http://genome.jgi-psf.org). These attributes make P. tricornutum a promising candidate for genetic modification.

In $P$. tricornutum, a stable nuclear transformation method is the only previously reported method for transformation (Niu et al. 2012; Apt et al. 1996b; Miyagawa et al. 2009). Plastids can be an ideal site of storage for the recombinant proteins compared to the cytoplasm, as adverse effects due to over accumulation can be avoided (Bogorad 2000). Plastid expression systems have been used successfully for the production of different biopolymers, therapeutic proteins and industrial enzymes. In plant cells, the presence of many transgene copies of the 
plastid genome per cell increases the accumulation of the foreign protein several folds, although the amount of the recombinant protein is mainly determined by factors such as transcriptional and posttranscriptional regulation of foreign genes, which determine the protein stability (De Marchis et al. 2012). For example, human somatotropin has been reported to accumulate at levels of up to $7 \%$ of the total soluble protein (TSP) when expressed in the plastid, which is 300 -fold greater than that of nuclear transformants (Staub et al. 2000). Furthermore, human serum albumin accumulated at levels up to $11.1 \%$ of the TSP, which is 500-fold higher than that of nuclear transformants (Millán et al. 2003). Additionally, plastid transformation has several advantages over nuclear transformation. For example, gene integration in the plastome occurs through homologous recombination, thus avoiding position effects; the high copy number of plastomes per cell and the lack of silencing machinery allow high and stable gene expression (Staub et al. 2000; McBride et al. 1995; Daniell et al. 1998; Sidorov et al. 1999). However, the success of plastid transformation largely depends on the species. Reproducible protocols are available for plastid transformation for some plant species (Maliga and Bock 2011). So far, there have been only few reports on transgene expression in the plastids of microalga, such as the green alga Chlamydomonas reinhardtii (Muto et al. 2009), the red alga Porphyridium sp. (Lapidot et al. 2002) and the euglenoid Euglena gracilis (Doetsch et al. 2001). Recently, a unique immunotoxin for cancer treatment has been successfully produced in Chlamydomonas plastids as a soluble protein with enzymatic activity, demonstrating a novel approach to the control of the disease (Tran et al. 2013). As the plastid genome of P. tricornutum was sequenced (Oudot-Le Secq et al. 2007), genetic manipulation of the plastid genome has attracted much attention. Genome mutations have been induced in the $p s b A$ gene in P. tricornutum plastids and consequently regulated photosynthesis (Materna et al. 2009). Here, we report the construction of a plastid expression vector and transformation system for overexpression of foreign genes in $P$. tricornutum plastids. Analysis of the plastid genome sequence of $P$. tricornutum revealed that it contains inverted repeat (IR) regions, distinct from the well-known microalgae Chlorella $\mathrm{sp}$. in which there is no IR region (Wakasugi et al. 1997). In this study, using the available plastid genome sequence for $P$. tricornutum, we have developed a high-efficiency plastid transformation system by introducing a specially designed TA cassette for cloning a heterologous gene between the two homologous recombination fragments trn $A$ and trnI from the IR regions of the plastid vector.

\section{Materials and Methods}

\section{Microalga Material Culture Conditions}

P. tricornutum was obtained from the Freshwater Algae Culture Collection, Institute of Hydrobiology, China (Cat. No. FACHB-863). The microalgae were grown as batch cultures in Erlenmeyer flasks containing $\mathrm{f} / 2$ medium, which was sterilised through $0.22-\mu \mathrm{m}$ filters (Millipore, Billerica, MA, USA). The cultures were grown at $21 \pm 1{ }^{\circ} \mathrm{C}$ in an artificial climate incubator. Cool-white fluorescent tubes provided an irradiance of $200 \mu \mathrm{mol}$ photons $\mathrm{m}^{-2} \mathrm{~s}^{-1}$ under long-day light conditions (15/9 h light/dark regime). In the PCR reactions, $P f u$ DNA polymerase was used to reduce the likelihood of introducing DNA mutations.

\section{Plasmid Construction for Plastid Transformation}

The plasmid pPtc-CAT (Fig. 1a), a plastid transformation vector, was constructed for testing the integration and expression of the CAT reporter gene in the plastid genome. It was generated by cloning the homologous recombination elements trnA/trnI and the CAT expression cassette into pMD19
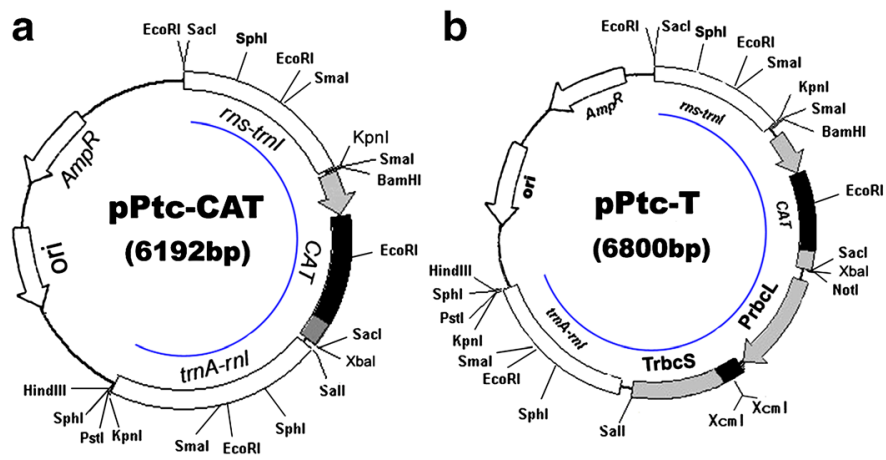

Fig. 1 Schematic maps showing plasmid constructs. a pPtc-CAT, b pPtc$\mathrm{T}$, $\mathbf{c}$ pPtc-eGFP and $\mathbf{d}$ map of plastid genome showing the integration sites. Annotations of the plasmid maps are given below: rns-trnI and trnA-rnl: The flanking regions derived from the P. tricornutum plastid genome used for homologous recombination during plastid transformation, CAT: reporter gene cassette encoding chloramphenicol acetyltransferase, PrbcL: promoter of the rubisco large subunit gene from

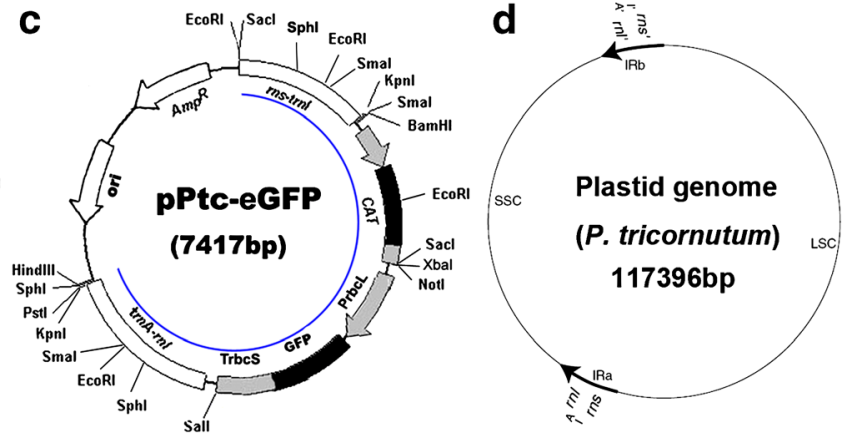

P. tricornutum and TrbcS: terminator of the rubisco small subunit gene from $P$. tricornutum; the CAT expression cassette was derived from Escherichia coli; eGFP: reporter gene encoding green fluorescent protein and $\mathrm{Amp}^{\mathrm{R}}$ : ampicillin resistance gene cassette from the plasmid pMD19. The arcs inside the vectors indicate the fragments recombining within the plastid genome. The map of plastid genome was derived from Oudot-Le Secq et al. (2007), where "I" and "A" indicate $\operatorname{trnI}$ and $\operatorname{trn} A$, respectively 
(TaKaRa, Dalian, China). The CAT expression cassette was derived from a bacterial source. Chloramphenicol acetyltransferase $(C A T)$ is a bacterial enzyme that detoxifies the antibiotic chloramphenicol, and it is responsible for chloramphenicol resistance. First, the total DNA from $P$. tricornutum was extracted using a Universal Genomic DNA Extraction Kit Ver.3.0 (TaKaRa, Dalian, China). Regions encompassing $1.1-\mathrm{kb}$ of $r n s-t r n I$ and $1.3-\mathrm{kb}$ of $\operatorname{trn} A-r n l$ in the $P$. tricornutum plastid genome were selected because they include homologous recombination elements. The fragments trnA-rnl and rns-trnI were cloned by PCR using total DNA as the template and primers, including P1 (5'-CGAGCTCCGAG CTCACTGGGCGTAAAGCGTCTGT-3' SacI site is underlined), P2 (5'-GGGGTACCGGGGTACCTTGGGCCA TTCTGGATTTG-3', KpnI site is underlined), P3 (5'ACGCGTCGACAACTGCAGCGGGGGTATAGCTCAG TTGG-3', SalI site is underlined) and P4 (5'-AACTGC AGACATGCATGCTTTCGTTACTCAAGCCGACATT-3', $\overline{P s t \mathrm{I}}$ site is underlined). The $S a c \mathrm{I}$ and $K p n \mathrm{I}$ restriction sites were added at the two ends of rns-trnI fragment for subcloning into the same sites in pMD19. Subsequently, the SalI and PstI sites flanking the trnA-rnl fragment were cloned using the same sites. Finally, the $C A T$ expression cassette was cloned by PCR using the plasmid pLysS (Novagen, USA) as a template with the following primers: P5 (5'-CGGGAT CCCGGGATCCAGCATCACCCGACGCACT-3', the Bam HI site is underlined) and P6 (5'-GCTCTAGAGCTCTA GATAACGACCCTGCCCTGAAC-3', the $X b a$ I site is underlined). The BamHI and $X b a \mathrm{I}$ sites flanking the $C A T$ expression cassette were cloned into the above plasmid harbouring the fragments trnA-rnl and rns-trnI, leading to the plastid transformation vector, pPtc-CAT.

To achieve a high-efficiency plastid vector for the cloning of target genes, the TA-cloning plasmid pPtc-T (Fig. 1b) was constructed. Initially, a target gene expression cassette of PrbcL-XcmI-XcmI-TrbcS was generated through the ligationindependent cloning (LIC) method. The PrbcL and TrbcS sequences are the promoter for the large subunit $(r b c L)$ and the terminator of the small subunit $(r b c S)$ of the rubisco genes, respectively. The 240-bp $\operatorname{PrbcL}$ fragment was amplified by PCR using total DNA as a template with the forward primer Pt358 (5'-gcTCTAGAGCGGCCGCAAACTTCTAAAACT TTCAATTAAAAGCTATTCT-3' the XbaI-NotI sites are underlined) and the reverse primer Pt347 (5'-TGATTTCT CCTTGGAATAAAAAGGCAATAT-3'). The PCR product was subsequently amplified by PCR with the forward primer Pt358 and the overlapped reverse primers Pt360a (5'-TCCT GGCCACCACAGGTGTGGTGATTTCTCCTTGGAATA AAAAGG-3' the XcmI site is underlined) and Pt360b (5'GTTTTTGTTCACCACCACTCTCCTGGCCACCACAGG TG-3', the $X c m$ I site is underlined). Thus the two $X c m$ I sites were added to the $3^{\prime}$ end of PrbcL. The 260-bp TrbcS fragment was cloned by PCR using total DNA as a template with the forward primer Pt344 (5'-TTTTTAGTAACAAAATAAAA TTAAAAAATGTTAAT-3') and the reverse primer Pt359 (5'-gcGTCGACCATTTTTTGTCATTGTGATAAGCTAA AGT-3', the SalI site is underlined). The PCR product was subsequently amplified by PCR with the forward primer Pt357Myc (5'-GAACAAAAACTCAGTGAAGAAGATCT TTAATTTTTAGTAACAAAATAAAATTAAA-3') and the reverse primer Pt359. Thus, the Myc-tagged sequence (encoding amino acids EQKLISEEDL) and stop codon were added upstream of TrbcS. The fragments PrbcL-XcmI-XcmI and Myc-TrbcS were ligated by LIC. The resulting fragment and plasmid pPtc-CAT were digested with SalI and XbaI and then ligated to a new vector pPtc-T for plastid transformation.

The gene of interest can be easily cloned into the vector pPtc-T using the TA cloning strategy. To generate the extra " $\mathrm{T}$ " at either 3 '-end of the linearised vector, it was predigested with $X c m \mathrm{I}$. To test the vector pPtc-T for target gene cloning and expression in the plastid, the eGFP (green fluorescent protein) reporter gene was cloned between PrbcL and TrbcS in the pPtc-T by TA cloning, generating the vector pPtc-eGFP (Fig. 1c). The $e G F P$ coding region was amplified with Taq polymerase to add an additional " $\mathrm{A}$ " at the 3 '-end using the primers eGFPf (5'-ACC ATGGTGAG CAAGGGCGAG GAGC TG-3') and eGFPr (5'-AACTCGAGCTTGTACAGCTC GTC-3'). Hence, the vector pPtc-eGFP utilises the plastid genomic sequences spanning the trnA-trnI region to target the heterologous gene into the plastid genome by homologous recombination (Fig. 1d).

\section{Transformation of $P$. tricornutum by Electroporation}

Plastid transformation was carried out by electroporation of P. tricornutum using a Bio-Rad Gene Pulser Xcell apparatus (Niu et al. 2012). Microalgae were aseptically spread on $\mathrm{f} / 2$ medium, supplemented with agar $(5 \mathrm{~g} / \mathrm{l})$ and chloramphenicol (30 g/l). Microalgal cells in the exponential phase were collected by centrifuging at $1,350 \mathrm{~g}$ for $10 \mathrm{~min}$. The pellets containing $0.5 \times 10^{7}$ microalgal cells were resuspended in $150 \mu 1.0 \mathrm{M} \mathrm{NaCl}$ then mixed with $150 \mu \mathrm{l} 0.1 \mathrm{M}$ mannitol and incubated in ice for $30 \mathrm{~min}$. Aliquots of $0.4 \mathrm{ml}$ were mixed with $0.4 \mu \mathrm{g}$ of the plasmid pPtc-eGFP and transferred into an electroporation cuvette (Gene Pulser/MicroPulser Cuvette, $0.4 \mathrm{~cm}$ gap, Bio-Rad). After electroporation, the cells were transferred to $10 \mathrm{ml}$ $\mathrm{f} / 2$ medium and incubated in the dark for $2 \mathrm{~h}$, followed by incubation for $24 \mathrm{~h}(12 \mathrm{~L}: 12 \mathrm{D})$. The cells were then collected by centrifugation at $1,500 \mathrm{~g}$ for $5 \mathrm{~min}$ and resuspended in $1 \mathrm{ml}$ medium. The cells were finally spread on $\mathrm{f} / 2$ selection medium supplemented with $5 \mathrm{~g} / \mathrm{l}$ agar containing various concentrations of chloramphenicol (200, 250 and $300 \mathrm{mg} / \mathrm{l})$. 
Analysis of the Transformed Microalgae

After 4 weeks of incubation of the plates under standard growth conditions, the putative transformed colonies were counted. The viable colonies were picked and inoculated into the fresh liquid $\mathrm{f} / 2$ medium containing $200 \mathrm{mg} / \mathrm{l}$ chloramphenicol and then subjected to five more subculture cycles on selection medium to obtain homoplastomic microalgae. To verify the plastid-transformed microalgae, the plastid DNA was extracted and used in PCR as a template. The plastids of $P$. tricornutum were isolated by sucrose gradient centrifugation. Cells in $50 \mathrm{ml}$ culture in the exponential phase were harvested by centrifugation at $5,000 \mathrm{~g}$ for $10 \mathrm{~min}$ at $4{ }^{\circ} \mathrm{C}$. The algal pellet was ground to a fine powder in liquid nitrogen and transferred to $1.5 \mathrm{ml}$ grinding buffer $(0.3 \mathrm{M}$ sucrose, $40 \mathrm{mM}$ Tris- $\mathrm{HCl}$ (pH 7.8), $5 \mathrm{mM} \mathrm{MgCl}_{2}, 1 \mathrm{mM}$ PMSF) followed by incubation on ice for $5 \mathrm{~min}$. The homogenate was subsequently separated by centrifugation at $350 \mathrm{~g}$ for $10 \mathrm{~min}$ at $4{ }^{\circ} \mathrm{C}$, and the pellet contained the nuclear fraction. The supernatant was carefully transferred to a new tube without disturbing the pellet, followed by centrifugation at $12,000 \mathrm{~g}$ for $20 \mathrm{~min}$ at $4{ }^{\circ} \mathrm{C}$, and the resultant pellet predominantly consisted of plastids. The plastid-rich fraction was confirmed by observation of chlorophyll fluorescence under a fluorescence microscope. The plastid DNA was extracted from the plastid-rich fraction using the Universal Genomic DNA Extraction Kit Ver.3.0 (TaKaRa).

To confirm the presence of the $\mathrm{CAT}$ gene in the microalgae, the cells were tested using PCR with the $C A T$-specific primers P5 and P6. Simultaneously, to validate the DNA integration and homologous recombination between trnA and trnI in the plastid genome in the transformed cells, PCR was performed with the primers IRf1 (5'-ATCGGCTAACTCCGTGCCAG$\left.3^{\prime}\right)$ or IRf1 (5'-TAACTATTTCTTATGACCTTTCC-3') and CATr (5'-AGCAACTGACTGAAATGCCTC-3'). The primer IRf1 and IRf2 were designed in the region of the $5^{\prime}$ upstream of the rns-trnI, which were not included in the cloned rns-trnI elements. The primer CATr was derived from the CAT coding region. The PCR reaction mixture $(25 \mu \mathrm{l})$ consisted of $250 \mathrm{ng}$ template, 5 pmol of each primer, $0.75 \mathrm{U}$ LA Taq polymerase (TaKaRa), $2.5 \mu 110 \times$ PCR reaction buffer, $1.5 \mu 1$ of $25 \mathrm{mM}$ $\mathrm{MgCl}_{2}$ and $0.25 \mu \mathrm{l}$ of each $10 \mathrm{mM}$ dNTP. PCR amplification was initiated with denaturation at $95^{\circ} \mathrm{C}$ for $3 \mathrm{~min}$, followed by 35 cycles of $95^{\circ} \mathrm{C} 1 \mathrm{~min}, 58{ }^{\circ} \mathrm{C} 1 \mathrm{~min}$ and $72{ }^{\circ} \mathrm{C} 1 \mathrm{~min}$. The expected PCR band on the gel was purified and analysed by sequencing.

Southern blot analysis was carried out to further confirm the gene integration as described previously (Manuell et al. 2007) by using the DIG DNA Labeling and Detection Kit (Roche, USA) following the manufacturer's instructions. The Universal Genomic DNA Extraction Kit Ver. 3.0 (TaKaRa) was used to extract the genomic DNA from the pPtc-eGFP transformed and untransformed control cells. Samples containing $5 \mu \mathrm{g}$ of genomic DNA were digested with EcoRI. The EcoRI/XbaI fragment in the coding region of CAT (Fig. 1) was used to prepare a DIG-labelled probe.

Transcription Levels of the CAT and eGFP Reporter Genes Quantified by Real-Time PCR

For quantification of the transcripts, the RNeasy Plant Mini kit (QIAGEN, USA) was used to extract the total RNA from $1 \times$ $10^{8} \mathrm{pPtc}-\mathrm{eGFP}$ transformed and untransformed P. tricornutum cells. During extraction, the RNA samples were treated with QIAGEN RNase-free DNase I to remove residualcontaminating DNA. In total, $2 \mu \mathrm{g}$ of total RNA was reverse transcribed with random hexamer primers using an Omniscript reverse transcription kit (QIAGEN). The RNA sample was used as template for cloning genes, while genes containing introns could be used as controls demonstrating complete DNA degradation. The primers for $C A T, e G F P$ and $\beta$-actin housekeeping marker were designed according to previous reports (De Riso et al. 2009; Niu et al. 2012). The qPCR was conducted in 96-well optical reaction plates using a SYBR Green Kit (Ruizhen Co., China) according to the manufacturer's instructions. Each sample was amplified in triplicate on a 7300 Sequence Detection System (Applied Biosystems, USA). The threshold cycle for each well was measured, and the relative transcription levels of the target gene compared with the control were quantified after normalisation to $\beta$-actin.

Protein Expression Determined by Western Blot and ELISA Analysis

The expression of the target gene could be rapidly tested through the Myc-tagged polypeptide present in the pPtc-T vector. To examine eGFP expression, a protein extraction kit (KeyGEN, Nanjin, China) was used to extract the total protein from $1 \times 10^{8}$ P. tricornutum cells transformed with pPtc-eGFP and an untransformed control. The protein concentrations were measured by the bicinchoninic acid assay (BCA assay). Twenty micrograms of protein from each sample was resolved by $12 \%$ SDS-PAGE at $100 \mathrm{~V}$ for $100 \mathrm{~min}$ and stained with Coomassie Brilliant Blue G-250 to visualise the protein bands. Two other identical gels were electrotransferred to a PVDF membrane. The membranes were blocked in phosphatebuffered saline and $0.1 \%$ Tween 20 (PBST) $(137 \mathrm{mM}$ $\mathrm{NaCl}, 2.7 \mathrm{mM} \mathrm{KCl}, 10 \mathrm{mM} \mathrm{Na} 2 \mathrm{HPO}_{4}, 1.8 \mathrm{mM} \mathrm{KH}_{2} \mathrm{PO}_{4}$, $0.5 \%$ Tween $20, \mathrm{pH} 7.6$ ) containing $5 \%$ bovine serum albumin (BSA) for $2 \mathrm{~h}$ at room temperature. After washing three times in PBST, the membranes were incubated with an anti-Myc antibody (Invitrogen, USA) or an anti-GFP antibody (Epitomics, USA) at a 1:5,000 dilution in $5 \%$ BSA in PBST for $2 \mathrm{~h}$ at room temperature. The anti-GAPDH and anti- $\beta$ actin antibodies (Abcam, UK) were used as internal controls at 
a dilution of 1:5,000. The membranes were then washed three times with PBST and incubated with a horseradish peroxidase (HRP)-conjugated secondary antibody (Kangwei, China) at a 1:5,000 dilution in PBST for $1 \mathrm{~h}$ at room temperature. The blots were washed three times and developed with tetramethyl-benzidine (TMB) reagent (Beyotime, China).

To quantify the algae-expressed recombinant eGFP protein, ELISA was conducted using an ELISA Kit (TSZ Elisa, Fuxing Bio., Shanghai, China). Briefly, an anti-GFP antibody was used to coat the microtiter plates. Then, the algal total soluble protein samples or GFP standards and a HRPconjugated secondary antibody were added, incubated and washed thoroughly. TMB substrate was added for the colour development, and the reaction was stopped using sulphuric acid. The eGFP was quantified according to the absorbance readings at OD450 using an ELISA reader (ELISA3000, DRG International Inc., NJ, USA) and the calculation from the eGFP standard curve.

\section{Laser-Scanning Confocal Microscopy}

A Zeiss LSM 510 Meta inverted confocal laser-scanning microscope (Zeiss, Jena, Germany) was used for the analysis of eGFP localisation as described by Li et al. (2008), using cells in the stationary phase. By means of multitracking, eGFP was excited at $488 \mathrm{~nm}$ and detected via a 505-530-nm band pass filter; chlorophyll fluorescence was excited at $543 \mathrm{~nm}$ and detected with a $600-\mathrm{nm}$ long-pass filter. The images were processed using the LSM 510 software (Zeiss, Jena, Germany).

\section{eGFP Expression Assayed by Flow Cytometry}

eGFP expression in the transformed cells was quantified by flow cytometry, which is considered a sensitive method (Ducrest et al. 2002). Cells $\left(1 \times 10^{4}\right)$ in the stationary phase were used for the eGFP fluorescence intensity assay. The cells were analysed using a FACS-Aria microflow cytometer (Becton Dickinson, NJ, USA). The dead cells and debris were eliminated using forward and side scatter parameters in the analysis. eGFP was excited at a wavelength of $488 \mathrm{~nm}$, and the fluorescence was detected with a 530/15-nm bandpass filter in the FL1 channel.

\section{Results}

Construction of Plastid Transformation Vectors

Schematic maps of the plastid transformation vectors pPtcCAT (Fig. 1a), pPtc-T (Fig. 1b) and pPtc-eGFP (Fig. 1c) are shown in Fig. 1. The homologous recombination elements rns-trnI and trnA-rnl from the P. tricornutum plastid genome were cloned into the plasmids. CAT and eGFP expression cassettes were inserted between the recombination elements. P. tricornutum was previously reported to be sensitive to chloramphenicol (Apt et al. 1996a, b), and we found that it could not survive $\geq 200 \mathrm{mg} / 1$ chloramphenicol treatment. Therefore, chloramphenicol was used as the selection marker for the expression vectors. The resultant plasmids pPtc-CAT, pPtc-T and pPtc-eGFP were 6,192, 6,800 and 7,417 bp, respectively. All the vectors were confirmed by restriction enzyme analysis and sequencing.

\section{Selection and Chloramphenicol Resistance of Plastid-Transformed Microalgae}

Transformed microalgae cells were selected on solid selection medium after electroporation, and putative transformed cells were streaked to purify and then cultured in liquid medium (Fig. 2). Transformed cells containing the plasmid pPtc-eGFPexpressing CAT grew well on the selection plates supplemented with $300 \mathrm{mg} / \mathrm{l}$ chloramphenicol (Fig. 2a), and these cells also grew well in the liquid medium supplemented with $300 \mathrm{mg} / \mathrm{l}$ chloramphenicol (Fig. 2b, right flask), whereas wild-type cells did not grow in the medium supplemented with $200 \mathrm{mg} / \mathrm{l}$ chloramphenicol (Fig. 2b, left flask). These results demonstrated that the heterologous $C A T$ gene was
Fig. 2 Plastid transformed microalgae. a Transformed cells expressing $C A T$ were determined on plates supplemented with $300 \mathrm{mg} / \mathrm{l}$ chloramphenicol; b surviving cells were picked from the plate and grown in liquid medium supplemented with $300 \mathrm{mg} / \mathrm{l}$ chloramphenicol (right flask), while wild-type cells could not grow in medium with $200 \mathrm{mg} / \mathrm{l}$ chloramphenicol (left flask)

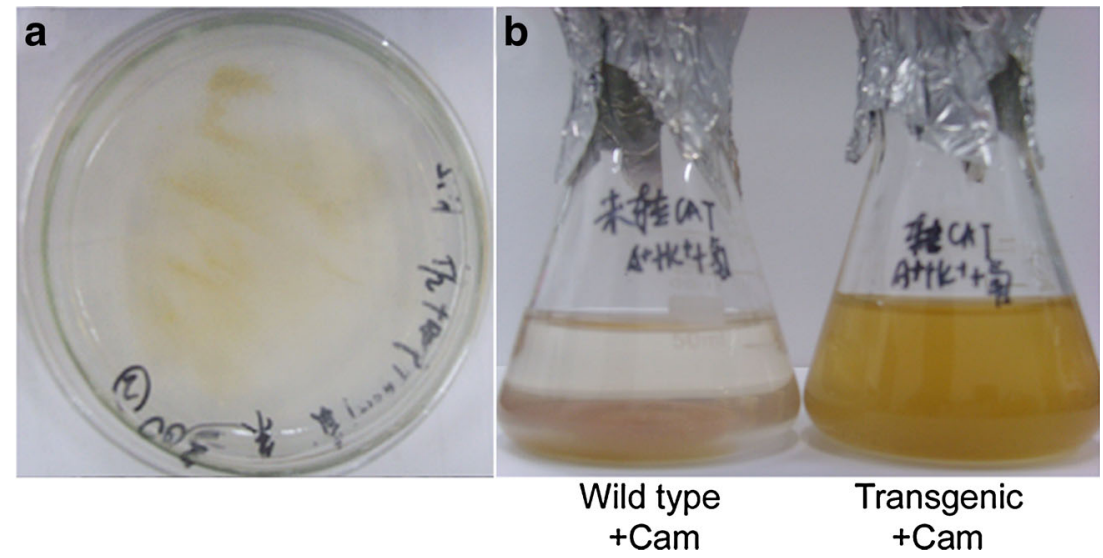


successfully expressed in the microalgae, and it exhibited antibiotic resistance. The overall efficiency of plastid transformation was estimated to be approximately one transplastomic colony per 1,000 microalgae cells $\left(5 \times 10^{3}\right.$ transformants in $5 \times$ $10^{6}$ cells per $0.4 \mu \mathrm{g}$ DNA), which is 100 -fold higher than that obtained with the $P$. tricornutum nuclear transformation system (Miyagawa et al. 2009; Apt et al. 1996a, b), indicating the occurrence of high-efficiency homologous recombination in the plastid genome.

\section{Culture and Analysis of Plastid-Transformed Microalgae}

Transformed microalgae cultured in liquid medium were subjected to PCR analysis using the CAT cassette primers P5 and P6 and another primer set including IRf and CATr. Gel electrophoresis showed a $1.1-\mathrm{kb}$ band as expected from the PCR with the P5 and P6 primers in the transformed microalgae (Fig. 3a, lane 2), but this band was absent in the wild type (Fig. 3a, lane 1). As expected, the 1.49 and 2.0-kb bands were obtained in the PCR with the IRf1+CATr and IRf2+CATr primers, respectively, in the transformed microalgae (Fig. 3b, lanes 2 and 5), but not in the wild type (Fig. 3b, lanes 1 and 4). The expected bands were purified and analysed by sequencing and showed the exact sequence including the rns-trnl on the transformation vector and the region on the plastid genome, indicating the integration of the $C A T$ cassette in the plastid genome of the microalgae by homologous recombination. The gene integration was further confirmed by Southern blot analysis. As shown in Supplemental Figure S1, a 2.9-kb hybridised band with the CAT probe was present in the transformed microalgae, whereas this band was not present in the wild type, demonstrating the integration of the CAT gene in the plastid genome. We observed that even the wildtype control showed two hybridised bands, which could be due to the similarity between the genomic sequences and the probe sequences used.

\section{Transcript Levels of the $C A T$ and $e G F P$ Reporter Genes Quantified by Real-Time PCR}

The relative transcript levels of the $C A T$ (Fig. 4a) and $e G F P$ (Fig. $4 \mathrm{~b}$ ) target genes compared with the control were quantified after normalisation to $\beta$-actin, and both of the reporters showed relatively high expression. Moreover, the transcript abundance of $e G F P$ was even higher, i.e., 33.34-fold, compared with $C A T$. This could be due to the advantages of the endogenous promoter for $e G F P$ over the bacterial promoter used in the CAT cassette.

\section{Western Blot and ELISA Analyses of the eGFP Reporter}

The expression of the $e G F P$ gene in the transformed cells was confirmed by Western blot analysis (Fig. 4c). Anti-Myc and anti-GFP antibodies were used as the probe for the Western blots, which showed a specific cross-reacting band with a molecular mass of $28 \mathrm{kDa}$ in the transformed cells, corresponding to the predicted size of eGFP, while there was no signal in the untransformed cells. This result confirmed the successful expression of the eGFP protein in the transformed cells using the plastid expression system in addition to demonstrating the feasibility of detecting recombinant fusion proteins by a Myc-tag.

To determine the quantity of the eGFP protein, the transformed microalgal cells were analysed by ELISA. Using an eGFP standard, the recombinant protein was shown to accumulate at a level up to $0.121 \%$ of the total soluble protein in the transformed microalgae (Fig. 4d).

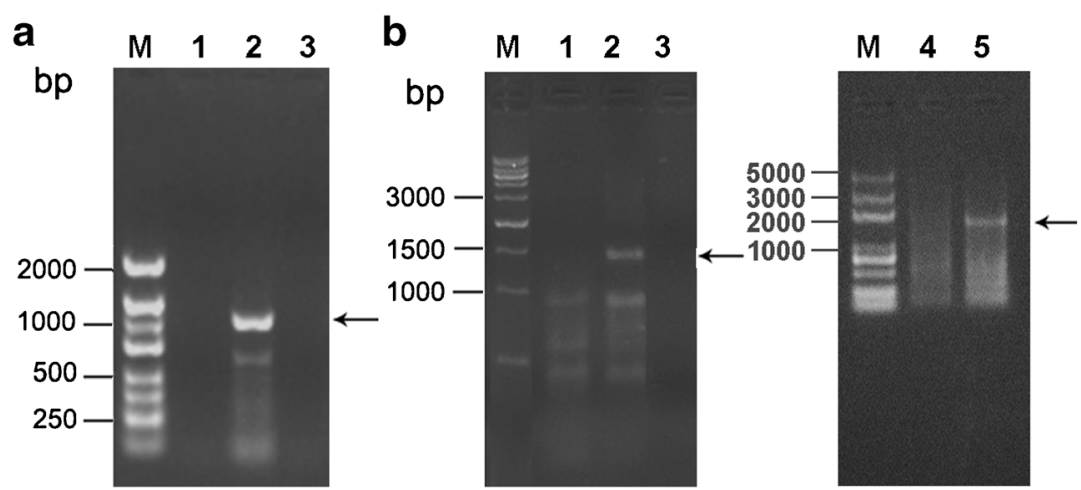

Fig. 3 Heterologous gene integration in plastid-transformed microalgae analysed by PCR. a Analysis of the CAT cassette using the primers P5 and $\mathrm{P} 6$ and $\mathbf{b}$ analysis of homologous recombination region using the primer pairs of IRf1 +CATr (left panel) and IRf2+CATr (right panel). Lane $M$ molecular mass markers, lane 1 PCR amplification of wild-type microalgae, lane 2 PCR amplification of transformed microalgae, lane
3 the negative control of the PCR amplification without a DNA template, lane 4 PCR amplification of wild-type microalgae and lane 5 PCR amplification of transformed microalgae; the arrows indicate the expected 1.1-kb CAT band, 1.49-kb IRf1+CATr and 2.0-kb IRf2+CATr homologous recombination region, respectively 
a

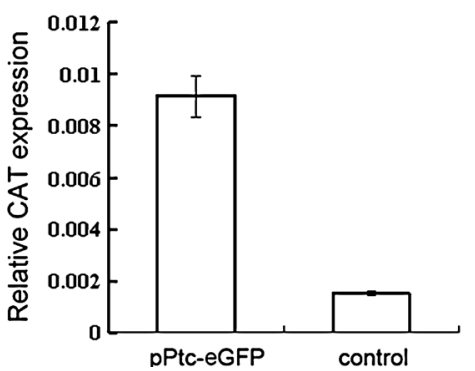

\section{b}

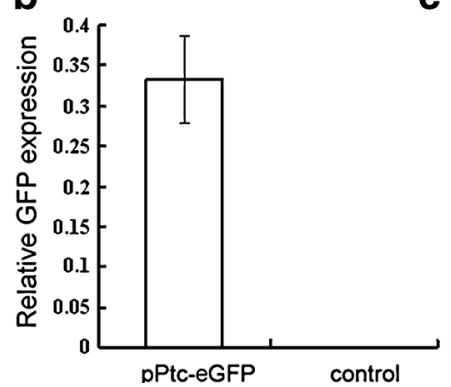

C

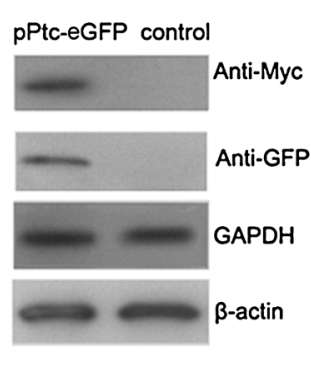

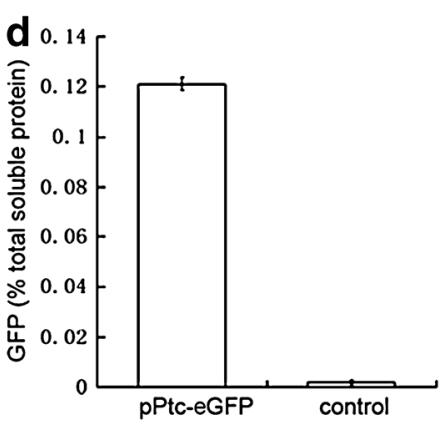

Fig. $4 \mathrm{mRNA}$ and protein expression of the reporter genes determined by qPCR, Western blot and ELISA analysis. pPtc-eGFP: transformed $P$. tricornutum and control: untransformed $P$. tricornutum. a $\mathrm{qPCR}$ of CAT expression. $\mathbf{b}$ qPCR of eGFP expression. $\mathbf{c}$ Western blot of eGFP expression. The proteins $(20 \mu \mathrm{g})$ were separated on $12 \%$ SDS-PAGE

\section{Plastid Localisation of eGFP in Transformed Microalgae}

The fluorescence of pPtc-eGFP transformed microalgae cells was examined using confocal microscopy. Strong green fluorescence was observed in the transformed cells in the green channel (Fig. 5a, a-1), while no fluorescence was detected in the untransformed cells (Fig. 5a, b-1). Moreover, the fluorescence was exclusively restricted to the plastids (Fig. 5a, a-4), which was indicated by the co-localisation of eGFP and autofluorescence of plastid due to chlorophyll. This result further confirmed the plastid-specific expression of the heterologous gene cloned in the newly derived vector pPtc-T. There was also a difference in cell morphology. The eGFPexpressing cells were thicker and shorter when compared to the control, whereas the control cells were long and spindleshaped (Fig. 5a, b). The results from the cytometry analysis showed high GFP fluorescence intensity in the eGFPexpressing cells, while wild-type cells showed only trace fluorescence caused by the overlap of emission spectra between GFP and algal autofluorescence (Fig. 5b). These results demonstrate that the eGFP reporter gene was successfully

gels, blotted to PVDF membranes and probed with antibodies. Anti-Myc and anti-GFP antibodies were used for eGFP detection; GAPDH and $\beta$ actin were used as internal controls. $\mathbf{d}$ eGFP accumulation was determined by ELISA

expressed at relatively high levels in the plastids. The growth speeds of the transformed and untransformed microalgae cultured in the liquid medium without chloramphenicol showed no visible difference, suggesting that the diatoms could tolerate the higher production of plastid encoded proteins.

\section{Discussion}

Heterologous gene expression in the nucleus in some of the microalgae species has been achieved with relatively low expression levels. It is imperative to develop a commercialscale microalgae bioreactor. Our study clearly demonstrates stable gene expression in the plastids of the diatom P. tricornutum, which could be a proof-of-principle plastid gene expression system for diatoms and an interesting tool to study diatom plastids.

This is the first plastid genetic transformation reported for the diatom based on an electroporation gene delivery mechanism, which is an economical and simple method. Electroporation has been widely used for the delivery of the a

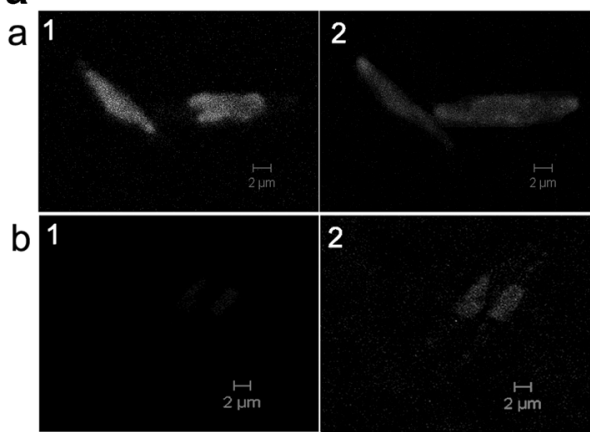

Fig. 5 eGFP expression in plastid-transformed P. tricornutum analysed by confocal microscopy and flow cytometry. a Confocal images showing eGFP expression. $a$ pPtc-eGFP transformed $P$. tricornutum, and $b$ untransformed P. tricornutum. 1 Green fluorescence of eGFP, 2 red fluorescence of the plastids, 3 DIC (differential interference contrast) and 4 overlay image indicating the co-localisation of green and red b
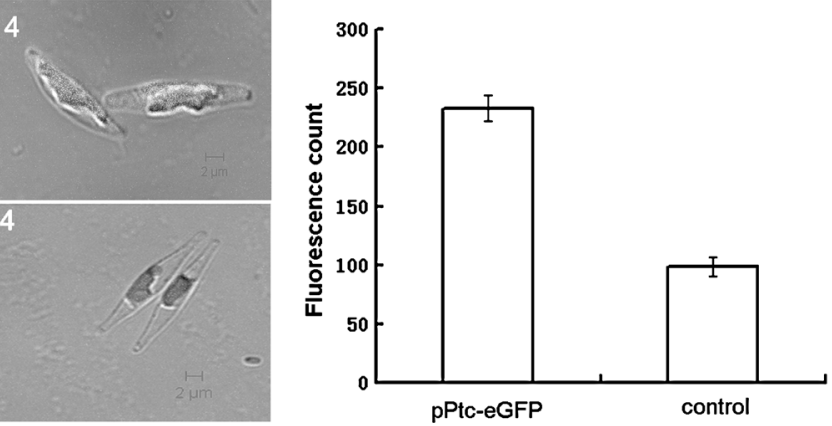

fluorescence. Bars $=2 \mu \mathrm{m}$. b The GFP fluorescence of triplicate samples was determined by flow cytometry, and their relative fluorescence intensities were calculated by subtracting the background counts. The left and right columns represent the control and pPtc-eGFP transformed cells, respectively. The images were taken with the same gain settings of the microscope 
DNA into microbes, plant cells and animal cells, but so far, there are only few reports in algae. Green algae species that have been successfully transformed by electroporation include C. reinhardtii (Brown et al. 1991) and Scenedesmus obliquus (Guo et al. 2013), with an efficiency of approximately 0.001 and $0.0005 \%$, respectively. To date, plastid transformation has solely relied on microprojectile bombardment using the Biolistic PDS-1000/He Particle Delivery System (Bio-Rad, USA). Plastid transformation in red alga Porphyridium species was also achieved using a gene gun system (Lapidot et al. 2002). Microprojectile bombardment is an expensive method in terms of both instrumentation and running cost, which limits its use in many laboratories. Our study using the electroporation technique could provide a rather low-cost and feasible way to transform microalgal plastids compared to the routine biolistic approach. The transformation efficiency was calculated to be $0.1 \%\left(5 \times 10^{3}\right.$ transformants in $5 \times 10^{6}$ cells per $0.4 \mu \mathrm{g}$ DNA), which is relatively higher than that reported for the red microcroalga Porphyridium sp., which was up to $2.5 \times 10^{-4}$ per $\mu \mathrm{g}$ DNA (Lapidot et al. 2002). Moreover, the high transformation efficiency makes this approach a highly promising tool for the genetic engineering of microalgae. Considering the possible presence of episomal plasmids in certain microalgae cells during the initial screening and subculture, it is necessary to examine the transformed microalgae using molecular approaches, such as Southern blotting, Northern blotting or Western blotting. The maintenance of the transplastomic lines can be achieved through long-term subculture without applying a selective pressure (Coll 2006).

A high-efficiency plastid transformation system was developed in this study, and this may be attributed to the selection of the specific homologous recombination sequences and also due to the presence of unique IR regions in the P. tricornutum plastid genome. The transgene is targeted to the particular IR, followed by the phenomenon of copy correction, which duplicates the introduced transgene into the other IR as well. This process causes transgenes to stably integrate at several sites within the plastid genome. Compared with transcriptionally silent spacer regions, transcriptionally active spacer regions offer unique advantages, such as the insertion of transgenes without $5^{\prime}$ or $3^{\prime}$ untranslated regions (UTRs) or promoters. The transcriptionally active intergenic region between the $\operatorname{trnI-trnA}$ genes within the rrn operon, existing in the IR region of the plastid genome, has been used for integration. High levels of expression of heterologous genes integrated at this site have been reported (Cosa et al. 2001). Plastid vectors may also carry an origin of replication that facilitates replication of the plasmid inside the plastid, thereby increasing the template copy number for homologous recombination and enhancing the probability of transgene integration (Verma and Daniell 2007). Moreover, the CAT reporter gene expressed in transformed P. tricornutum was sufficient to confer resistance to chloramphenicol, which contributed to the effective selection of the transgenic microalgae.

Generally, the progress of biotechnology/genetic engineering in microalgae has been slow when compared to other systems. In the case of P. tricornutum, the transformation efficiencies reported to date were even lower than those for other pennate diatoms, such as Cylindrotheca fusiformis, and the centric diatom Thalassiosira pseudonana (Poulsen et al. 2006; Poulsen and Kröger 2005). In this study, we used the $r b c L$ promoter from the $P$. tricornutum plastid genome. The $r b c L$ promoter is considered to be a strong and constitutive promoter, and it has been widely used in plastid expression vector systems (Verma and Daniell 2007). Additionally, a new approach was employed for easy cloning of the gene of interest between the promoter and terminator in the plasmid pPtc-T when compared to the other commonly used vectors. The two specially designed adjacent $X c m \mathrm{I}$ sites created an extra " $\mathrm{T}$ " at both 3'ends of the vector upon $X c m I$ digestion, which allows for the efficient cloning of any gene into the $P$. tricornutum plastid via the TA system. The Myc-tag in the vector provides a tool for testing and purifying the recombinant fusion protein with commercially available reagents to study the heterologous genes expressed in the P. tricornutum plastid. Our study shows that the expression level of eGFP was relatively high in the transformed P. tricornutum compared with the plastid expression in C. reinhardtii, where eGFP without codon optimisation accumulated $\sim 0.006 \%$ of total soluble protein (Franklin et al. 2002). Considering the profound effect of codon usage on the expression of heterologous proteins in microalgae plastids (Mayfield et al. 2007), the current $P$. tricornutum plastid expression system would be expected to have the potential of a much higher protein yield for codon-optimised genes. Interestingly, the morphology of the transformed cells became egg-shaped from the original fusiform, and the size was also smaller than that of the untransformed wild-type cells. Genetic engineering of P. tricornutum will be particularly useful for the industrial production of valueadded proteins and also to improve its nutritional value, as it is widely used as the live feed in the aquaculture industry.

Acknowledgements This work was supported by the National Technologies Program (2011BAD14B03), Science and Technology Project of Guangdong (2010B030600005, 2009B050600005).

Open Access This article is distributed under the terms of the Creative Commons Attribution License which permits any use, distribution, and reproduction in any medium, provided the original author(s) and the source are credited.

\section{References}

Apt KE, Kroth-Pancic PG, Grossman AR (1996a) Stable nuclear transformation of the diatom Phaeodactylum tricornutum. Mol Gen Genet 252:8 
Apt KE, Kroth-Pancic PG, Grossman AR (1996b) Stable nuclear transformation of the diatom Phaeodactylum tricornutum. Mol Gen Genet 252:572-579

Bogorad L (2000) Engineering chloroplasts: an alternative site for foreign genes, proteins, reactions and products. Trends Biotechnol 18:257263

Brown LE, Sprecher SL, Keller LR (1991) Introduction of exogenous DNA into Chlamydomonas reinhardtii by electroporation. Mol Cell Biol 11:5

Coll JM (2006) Review. Methodologies for transferring DNA into eukaryotic microalgae. Span J Agric Res 4:316-330

Cosa BD, Moar W, Lee S-B, Miller M, Daniell H (2001) Overexpression of the $\mathrm{Bt}$ cry2Aa2 operon in chloroplasts leads to formation of insecticidal crystals. Nat Biotechnol 19:4

Daniell H, Datta R, Varma S, Gray S, Lee SB (1998) Containment of herbicide resistance through genetic engineering of the chloroplast genome. Nat Biotechnol 16:345-348

De Marchis F, Pompa A, Bellucci M (2012) Plastid proteostasis and heterologous protein accumulation in transplastomic plants. Plant Physiol 160:571-581

De Riso V, Raniello R, Maumus F, Rogato A, Bowler C, Falciatore A (2009) Gene silencing in the marine diatom Phaeodactylum tricornutum. Nucleic Acids Res 37:e96

Doetsch NA, Favreau MR, Kuscuoglu N, Thompson MD, Hallick RB (2001) Chloroplast transformation in Euglena gracilis: splicing of a group III twintron transcribed from a transgenic psbK operon. Curr Genet 39:49-60

Ducrest AL, Amacker M, Lingner J, Nabholz M (2002) Detection of promoter activity by flow cytometric analysis of GFP reporter expression. Nucleic Acids Res 30:e65

Franklin S, Ngo B, Efuet E, Mayfield SP (2002) Development of a GFP reporter gene for Chlamydomonas reinhardtii chloroplast. Plant J 30:733-744

Guo SL, Zhao XQ, Tang Y, Alam MA, Wan HSH, Bai FW, Chang JS (2013) Establishment of an efficient genetic transformation system in Scenedesmus obliquus. J Biotechnol 163:61-68

Lapidot M, Raveh D, Sivan A, Arad SM, Shapira M (2002) Stable chloroplast transformation of the unicellular red alga Porphyridium species. Plant Physiol 129:7-12

Li HY, Xiao S, Chye ML (2008) Ethylene- and pathogen-inducible Arabidopsis acyl-CoA-binding protein 4 interacts with an ethylene-responsive element binding protein. J Exp Bot 59:39974006

Maliga P, Bock R (2011) Plastid biotechnology: food, fuel, and medicine for the 21st century. Plant Physiol 155:1501-1510

Manuell AL, Beligni MV, Elder JH, Siefker DT, Tran M, Weber A, McDonald TL, Mayfield SP (2007) Robust expression of a bioactive mammalian protein in Chlamydomonas chloroplast. Plant Biotechnol J 5:402-412

Materna AC, Sturm S, Kroth PG, Lavaud J (2009) First induced plastid genome mutations in an alga with secondray plastids: psbA mutations in the diatom Phaeodactylum tricornutum (Bacillariophyceae) reveal consequences on the regulation of photosynthesis. J Phycol $45: 838-846$
Mayfield SP, Manuell AL, Chen S, Wu J, Tran M, Siefker D, Muto M, Marin-Navarro J (2007) Chlamydomonas reinhardtii chloroplasts as protein factories. Curr Opin Biotechnol 18: 126-133

McBride KE, Svab Z, Schaaf DJ, Hogan PS, Stalker DM, Maliga P (1995) Amplification of a chimeric Bacillus gene in chloroplasts leads to an extraordinary level of an insecticidal protein in tobacco. Biotechnology (NY) 13:362-365

Millán AF-S, Mingo-Castel A, Miller M, Daniell H (2003) A chloroplast transgenic approach to hyper-express and purify human serum albumin, a protein highly susceptible to proteolytic degradation. Plant Biotechnol J 1:71-79

Miyagawa A, Okami T, Kira N, Yamaguchi H, Ohnishi K, Adachi M (2009) Research note: high efficiency transformation of the diatomPhaeodactylum tricornutumwith a promoter from the diatom Cylindrotheca fusiformis. Phycol Res 57:142-146

Muto M, Henry RE, Mayfield SP (2009) Accumulation and processing of a recombinant protein designed as a cleavable fusion to the endogenous Rubisco LSU protein in Chlamydomonas chloroplast. BMC Biotechnol 9:26

Niu YF, Yang ZK, Zhang MH, Zhu CC, Yang WD, Liu JS, Li HY (2012) Transformation of diatom Phaeodactylum tricornutum by electroporation and establishment of inducible selection marker. Biotechniques. doi:10.2144/000113881

Oudot-Le Secq MP, Grimwood J, Shapiro H, Armbrust V, Bowler C, Green BR (2007) Chloroplast genomes of the diatoms Phaeodactylum tricornutum and Thalassiosira pseudonana: comparison with other plastid genomes of the red lineage. Mol Genet Genomics 277:427-439

Poulsen N, Kröger N (2005) A new molecular tool for transgenic diatoms. FEBS J 272:3413-3423

Poulsen N, Chesley PM, Kröger N (2006) Molecular genetic manipulation of the diatom Thalassiosira pseudonana (Bacillariophyceae). J Phycol 421059-1065

Sidorov VA, Kasten D, Pang SZ, Hajdukiewicz PTJ, Staub JM, Nehra NS (1999) Stable chloroplast transformation in potato: use of green fluorescent protein as a plastid marker. Plant $\mathrm{J}$ 19:209-216

Staub JM, Garcia B, Graves J, Hajdukiewicz PT, Hunter P, Nehra N, Paradkar V, Schlittler M, Carroll JA, Spatola L, Ward D, Ye G, Russell DA (2000) High-yield production of a human therapeutic protein in tobacco chloroplasts. Nat Biotechnol $18: 333-338$

Tran M, Van C, Barrera DJ, Pettersson PL, Peinado CD, Bui J, Mayfield SP (2013) Production of unique immunotoxin cancer therapeutics in algal chloroplasts. Proc Natl Acad Sci U S A 110:E15-E22

Verma D, Daniell H (2007) Chloroplast vector systems for biotechnology applications. Plant Physiol 145:1129-1143

Wakasugi T, Nagai T, Kapoor M, Sugita M, Ito M, Ito S, Tsudzuki J, Nakashima K, Tsudzuki T, Suzuki Y, Hamada A, Ohta T, Inamura A, Yoshinaga K, Sugiura M (1997) Complete nucleotide sequence of the chloroplast genome from the green alga Chlorella vulgaris: the existence of genes possibly involved in chloroplast division. Proc Natl Acad Sci U S A 94:5967-5972 